\title{
High-grade soft tissue sarcomas of the extremities: surgical margins influence only local recurrence not overall survival
}

\author{
Julie Willeumier • Marta Fiocco • Remi Nout • Sander Dijkstra • \\ William Aston • Rob Pollock • Henk Hartgrink • Judith Bovée • \\ Michiel van de Sande
}

Received: 10 January 2015 / Accepted: 26 January 2015 / Published online: 7 March 2015

(C) The Author(s) 2015. This article is published with open access at Springerlink.com

\begin{abstract}
Purpose After surgical treatment of high-grade soft tissue sarcomas, local recurrences, metastases and survival remain a great concern. Further knowledge on factors with a possible impact on these endpoints, specifically resection margins, is relevant for decision-making regarding the aggressiveness of local treatment. The aim of this study is to investigate the impact of prognostic factors on local recurrence and overall survival for patients with high-grade soft tissue sarcomas of the extremities.

Methods In a retrospective cohort study of 127 patients (mean age 48 years, range five to 91 ; median follow-up 71 months) the prognostic effect of margin status and other clinicopatho-
\end{abstract}

J. Willeumier $(\varangle) \cdot$ S. Dijkstra $\cdot$ M. van de Sande

Department of Orthopaedic Surgery, Leiden University Medical

Center, Leiden, The Netherlands

e-mail: j.j.willeumier@lumc.nl

\section{Fiocco}

Department of Medical Statistics and Bioinformatics,

Leiden University Medical Center, Leiden, The Netherlands

\section{Fiocco}

Mathematical Institute, Leiden University, Leiden, The Netherlands

R. Nout

Department of Clinical Oncology and Radiotherapy,

Leiden University Medical Center, Leiden, The Netherlands

W. Aston $\cdot$ R. Pollock

Department of Orthopaedic Oncology, Royal National

Orthopaedic Hospital, Stanmore, UK

H. Hartgrink

Department of Surgical Oncology, Leiden University

Medical Center, Leiden, The Netherlands

J. Bovée

Department of Pathology, Leiden University

Medical Center, Leiden, The Netherlands logic characteristics on local recurrence and overall survival were analysed by employing a multivariate Cox regression. Results Five-year cumulative incidence of local recurrence and distant metastases was $26 \%$ and $40 \%$, respectively. The estimated five-year overall survival was $59 \%$. Tumour size proved a consistent adverse prognostic factor for local recurrence (hazard ratio (HR) 3.9), distant metastasis (HR 4.9) and overall survival (HR 2.4). The significant association of resection margins with local recurrence (HR 10.2) was confirmed. Margins were however not significantly associated with the occurrence of distant metastasis or overall survival. The occurrence of local recurrence had a significant impact on overall survival (HR 2.0).

Conclusions The results of this study confirm the critical role of tumour size on survival and margins on local recurrence, and stress the need for further investigation concerning the association between margins, local recurrence and survival.

Keywords Soft tissue sarcoma - Treatment · Margins · Prognosis $\cdot$ Survival

\section{Introduction}

Soft tissue sarcomas (STS) are a rare (incidence 1:30.000) heterogeneous group of tumours accounting for approximately $1 \%$ of all adult cancers [1]. Despite continuous improvements in imaging, surgical techniques and adjuvant treatment, local recurrences, distant metastasis and death remain of great concern especially in high-grade tumours. Five-year cumulative incidence rates range from 12 to $28 \%$ for local recurrence and $21-40 \%$ for distant metastasis [2-6]. Defining a consistent set of prognostic variables for these oncologic end-points has proven challenging due to the heterogeneity and low incidence of high-grade tumours $(\approx 38 \%$ of all STS) [7]. Some factors have consistently proven to be predictive of adverse 
events, such as histotype, depth, histological grade, and size [2-11]. The role of resection margins has also been extensively studied in highly heterogeneous sarcoma populations. There is general agreement that positive margins are associated with a higher risk of local recurrence [4-6, 8-11]. The association between resection margins and distant metastasis or overall survival however remains unclear [3, 4, 6, 8-11]. The majority of studies addressing this issue have included all histologic grades and sarcoma types leading to general outcomes, which are intricate to use in a clinical setting. Questioning the effect of margins and local recurrences on survival remains relevant, as it influences the extent of local and adjuvant treatment. This study focuses only on STS of high grade to improve understanding of this subgroup of unfavourable STS that is more homogeneous in the context of survival outcomes.

A competing risks model was used to estimate the cumulative incidence of local recurrence and distant metastasis. When the situation is such that the occurrence of an event (e.g., death) prevents the event of interest (e.g., local recurrence) from happening, a competing risks model must be applied [12]. If this analysis is not used, the cumulative incidence rate (the probability of failure from a specific cause) is generally overestimated.

The objective of this study was to evaluate the effect of margins, adjusted for other known risk factors, on local recurrence, distant metastases, and overall survival.

\section{Patients and methods}

A retrospective analysis of patients surgically treated between 2000 and 2007 at our two tertiary referral centres for primary, high-grade, STS of the extremities (ESTS) was performed. From the pathology databases 161 patients with one of four most common high-grade ESTS diagnoses-high-grade myxofibrosarcoma, synovial sarcoma, malignant peripheral nerve sheath tumour, or angiosarcoma-were identified. Exclusion criteria were metastatic disease at time of diagnosis, presentation with recurrent disease, no operative treatment with curative intent at our institutions, and an unknown margin status. All patients had pre-treatment staging with a lung CT-scan to rule out the presence of metastasis. Thirty-four patients were excluded from analysis (presentation with metastases (11), recurrent disease (16), no operative treatment (7)) resulting in a cohort of 127 patients (mean age at time of diagnosis: 48 years (range five to 91), including nine children; male/female ratio 1:0.87).

Medical records including operative and pathologic reports were reviewed and the following information was recorded: age, gender, presentation status (no treatment/biopsy only vs. prior (Whoops) excision), tumour size (small $(<50 \mathrm{~mm})$ vs. intermediate $(50-100 \mathrm{~mm})$ vs. large $(\geq 100 \mathrm{~mm})[8])$, depth (superficial vs. deep to investing fascia), location (upper vs. lower extremity), involvement of adjacent structures, surgical margin, adjuvant therapy (radiotherapy vs. no radiotherapy; chemotherapy vs. no chemotherapy), and follow-up data. Table 1 summarizes patient, tumour and treatment characteristics.

Independent experienced musculoskeletal pathologists ( $\mathrm{PH}, \mathrm{JB}, \mathrm{AF}$ ) defined the closest surgical margin after inking and sectioning of the specimen. The closest identified resection margin was categorized as follows: intralesional for a positive margin with tumour cells visible at the inked margin (12\%), marginal for a margin of $2 \mathrm{~mm}$ or less $(58 \%)$, or wide for a negative margin of more than $2 \mathrm{~mm}$ (30\%) [13]. The decision concerning the use of adjuvant treatment was not uniform during the study period due to progressing insights in the lapse of time, although nearly all patients $(80 \%)$ received radiotherapy. The most common regimen was $50 \mathrm{~Gy}$ on the target region and a boost of 10Gy on the tumour region. Adjuvant therapy was omitted in $24(19 \%)$ patients for various reasons (amputation, patient preference (seven patients), superficial tumours (seven tumours) or wide resection margins (12 patients)). No other adjuvant therapies, besides chemotherapy or radiotherapy, were administered.

Local recurrence was defined as the first clinical, radiological or pathological manifestation of tumour of the same histologic type within or contiguous to the previously treated tumour bed, one or more months after primary treatment. Distant recurrence was defined by clinical or radiological evident systemic spread of tumour outside the primary tumour bed, including nodal metastasis. Dates of death were extracted from the medical records, which were coupled to the general registry of the hospital.

\section{Statistical analysis}

The outcomes of interest were five year cumulative incidence of local recurrence and distant metastasis, and five year overall survival rate. Synchronous local and distant recurrences were considered as separate events in the analysis of local recurrence and distant metastasis. Time to recurrence and time to death were estimated from date of first surgery (excluding previous Whoops excisions). Survival end-point was date of last follow-up or death. The median follow-up, as assessed by employing the reverse Kaplan-Meier method [14], was 71 months (95\% CI 62-80).

The following variables were tested for univariate association with local-recurrence, distant-recurrence and survival using the log rank test statistic: age, gender, anatomic site, tumour growth, depth, previous Whoops excision, planned primary amputation, margin, histological subtype, and adjuvant radio- or chemotherapy. Factors identified as significant were included in the multivariate analysis. For local recurrence and distant metastasis a cause-specific multivariate 
Table 1 Patient, tumour and treatment characteristics of high-grade soft tissue sarcomas of the extremities

\begin{tabular}{|c|c|c|}
\hline Characteristic & Number & Percent $(\%)$ \\
\hline \multicolumn{3}{|l|}{ Gender } \\
\hline Male & 68 & 63.5 \\
\hline Female & 59 & 46.5 \\
\hline \multicolumn{3}{|l|}{ Primary presentation } \\
\hline No treatment/biopsy only & 102 & 80.3 \\
\hline Prior excision & 25 & 19.7 \\
\hline \multicolumn{3}{|l|}{ Tumour location } \\
\hline Upper extremity & 25 & 19.7 \\
\hline Lower extremity & 102 & 80.3 \\
\hline \multicolumn{3}{|l|}{ Tumour size } \\
\hline$<50 \mathrm{~mm}$ & 36 & 28.3 \\
\hline $50-99.9 \mathrm{~mm}$ & 44 & 34.6 \\
\hline$\geq 100 \mathrm{~mm}$ & 42 & 33.1 \\
\hline Unknown & 5 & 3.9 \\
\hline \multicolumn{3}{|l|}{ Depth $^{\mathrm{a}}$} \\
\hline Superficial & 17 & 13.4 \\
\hline Deep & 104 & 81.9 \\
\hline Unknown & 7 & 4.7 \\
\hline \multicolumn{3}{|l|}{ Histopathology } \\
\hline Angiosarcoma & 5 & 3.9 \\
\hline MPNST & 27 & 21.3 \\
\hline Myxofibrosarcoma & 46 & 36.2 \\
\hline Synovial sarcoma & 45 & 35.4 \\
\hline Sarcoma nos & 4 & 3.1 \\
\hline \multicolumn{3}{|l|}{ Surgical margin } \\
\hline Intralesional & 15 & 11.8 \\
\hline Marginal 0-2 mm & 74 & 58.3 \\
\hline Wide $>2 \mathrm{~mm}$ & 38 & 29.9 \\
\hline \multicolumn{3}{|l|}{ Type of surgery } \\
\hline Limb-sparing & 121 & 95.3 \\
\hline Amputation & 6 & 4.7 \\
\hline \multicolumn{3}{|l|}{ Adjuvant therapy } \\
\hline Yes & 103 & 81.1 \\
\hline No & 6 & 4.7 \\
\hline Unknown & 18 & 14.2 \\
\hline \multicolumn{3}{|l|}{ Radiotherapy } \\
\hline Yes & 96 & 79.6 \\
\hline No & 31 & 20.4 \\
\hline \multicolumn{3}{|l|}{ Chemotherapy } \\
\hline Yes & 24 & 18.9 \\
\hline No & 103 & 81.1 \\
\hline
\end{tabular}

MPNST malignant peripheral nerve sheath tumour, Sarcoma nos sarcoma not otherwise specified

${ }^{a}$ Depth: relative to the investing fascia

hazard Cox regression model was applied. The cause-specific hazard function is the fundamental concept in the competing risks model; it provides the hazard of failing from a specific cause in the presence of the competing events [12]. For overall survival a Cox model with local and distant recurrences as time-dependent covariates was estimated. $P$ values at or below 0.05 were considered significant. To check the proportional hazards assumption we looked at the Shoenfeld residuals. Statistical analyses were performed in SPSS (version 20; IBM Corp, Armonk, NY) and R 2-18 [15]. All analyses concerning competing risks were performed with the MState library [16].

\section{Results}

Disease outcome and patient survival

Five year cumulative incidence of local recurrence and distant metastases was $26 \%$ and $40 \%$, respectively. The estimated five-year overall survival was $59 \%$ with a median survival time of 79 months (95\% CI, 65-93 months) (Fig. 1).

Among patients with intralesional margins, six (50\%) and seven $(58 \%)$ patients developed a local recurrence and/or distant metastasis, respectively. This was the case for 22 (30\%) and $32(43 \%)$ patients with marginal margins, and three (8\%) and $11(29 \%)$ patients with wide margins. Eventually, seven (47\%), 37 (50\%), and 14 (47\%) patients with intralesional, marginal, or wide margins, respectively, died.

Prognostic factors

Table 2 shows the results from the univariate and multivariate analyses for all endpoints.

Univariate prognostic factors for local recurrence were margin status, tumour size, and age. For distant metastases, tumour size and a presentation after 'Whoops' surgery were significant factors. Tumour size, age and a primary limb sparing operation (vs. amputation) were significant factors for overall survival. Radiotherapy did not emerge as a significant univariate prognostic factor for either endpoint; merely a slight trend towards better survival was observed.

In the multivariate analysis, prognostic factors for local recurrence were margins and tumour size. Patients with intralesional and marginal resection margins had 10.2 times (95\% CI, 2.5-42.4) and 4.8 times (95\% CI, 1.4-16.2) higher risk (95\% CI, 1.4-16.2) of experiencing local recurrence, respectively. Patients presenting with large tumours ( $\geq 100 \mathrm{~mm}$ ) had 3.9 times higher risk (95\% CI, 1.5-10.4) of developing a local recurrence compared to patients with small $(<50 \mathrm{~mm})$ tumours. In Figs. 2 and 3 the cumulative incidence curves of local recurrence are represented for resection margin and tumour size respectively. For distant metastases, tumour size remained the only prognostic factor associated with the occurrence of distant metastases (hazard ratio 4.9 (95\% CI, 1.9-12.4) and 2.4 (95\% CI, 0.9-6.2)) for large and intermediate tumours, respectively). No correlation between tumour 
Fig. 1 Overall survival

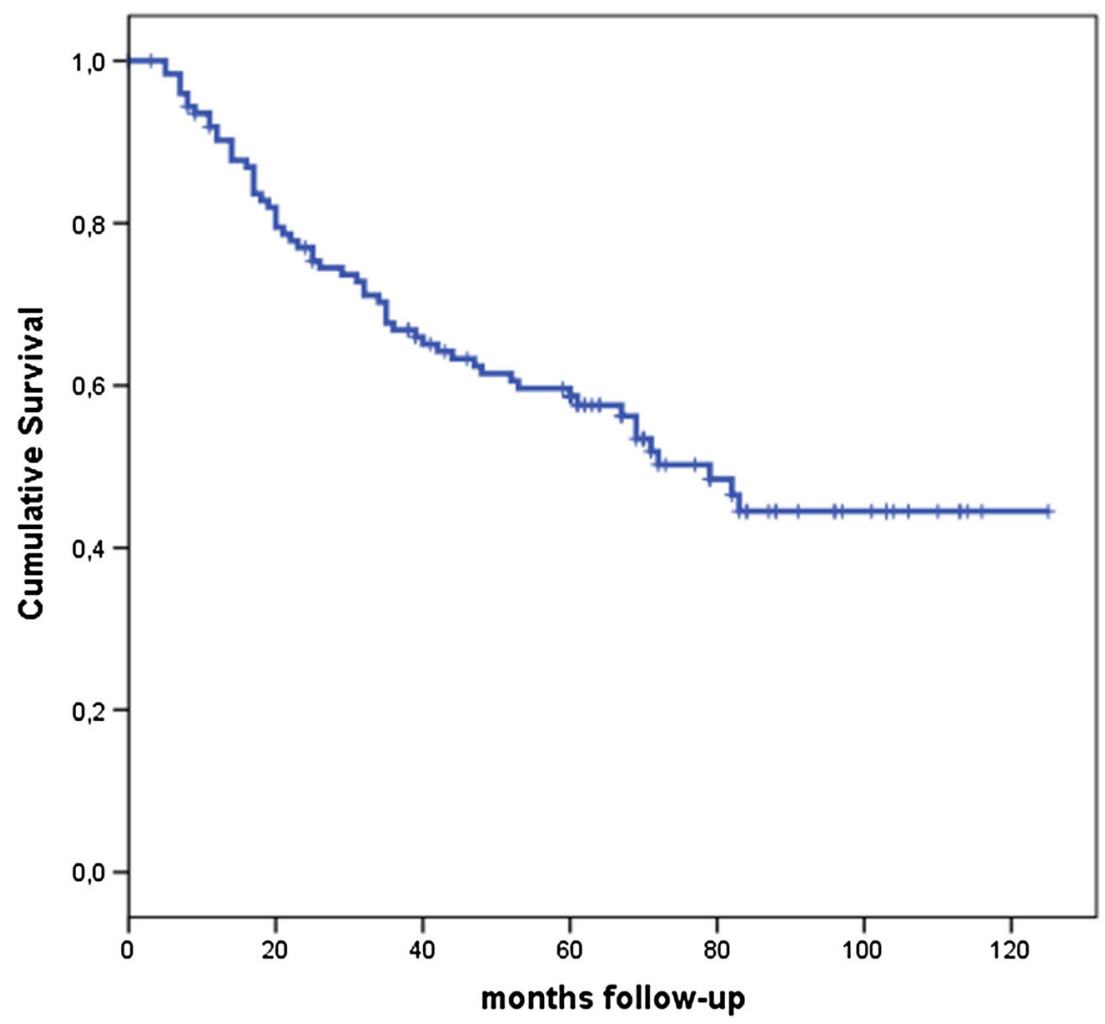

size and surgical margins could be observed (Table 3). To assess the impact of risk factors on overall survival a Cox model with local recurrence as time-dependent variable has been estimated. Independent significant factors were size and presence of local recurrence. The hazard ratio for tumour size was equal to 2.4 (95\% CI, 1.1-2.9) and 1.1 (95\% CI, 0.5-2.5) for patients with large or intermediate tumours, respectively, compared to patients with small tumours. Development of a local recurrence was associated with a 2.0-fold (95\% CI, 1.13.7) increased risk of death. A Cox model with distant metastasis as time-dependent covariate was also employed. As expected the hazard ratio associated with distant metastasis was extremely high, outweighing all other prognostic variables. As a result none showed a significant statistical effect. The risk of death increased 17-fold with a distant metastasis $(95 \% \mathrm{CI}$, 8.6-33.3).

\section{Discussion}

Surgery remains the cornerstone of treatment for patients with ESTS. Although local control is one of the primary objectives of treatment, the question is whether this has to be sought for at every extent, leading to possibly unacceptable functional outcomes. The lack of unambiguous evidence that margins and local control influence survival further complicates decision-making. Moreover, results from many studies are confounded by the amount of histological subtypes included. Therefore this study aimed to define the influence of the extent of local treatment (i.e., margins) in the light of other prognostic factors on local recurrence and survival in a specific group of high-grade ESTS.

The cumulative incidences of local recurrence and distant metastases at five years were $26 \%$ and $40 \%$, respectively. Five-year overall survival was $59 \%$. These results are somewhat less optimistic than those previously published, although a comparison is difficult due to differences in the patient population (high grade vs. all STS subtypes) and statistical methods $[2,17,18]$. As expected, tumour size was a consistent adverse prognostic risk factor for the occurrence of local recurrence, distant metastasis, and overall survival, in agreement with published results [4, 6, 11, 19].

In this series, intralesional and marginal margins had a significant effect on the development of local recurrences, while the development of distant metastases and overall survival were not significantly affected by the surgical margin. This apparent controversial manifestation has been reported previously, although a clear explanation is still lacking. Generally it is hypothesized that these results can be explained on the basis of tumour-specific or biologic factors, with highly aggressive tumours leading to greater risk of distant spread and subsequent death irrespective of local surgery [4, 8, 9, $17,20]$. Following from this, the question of the prognostic role of local recurrence arises. In this study, the occurrence of 


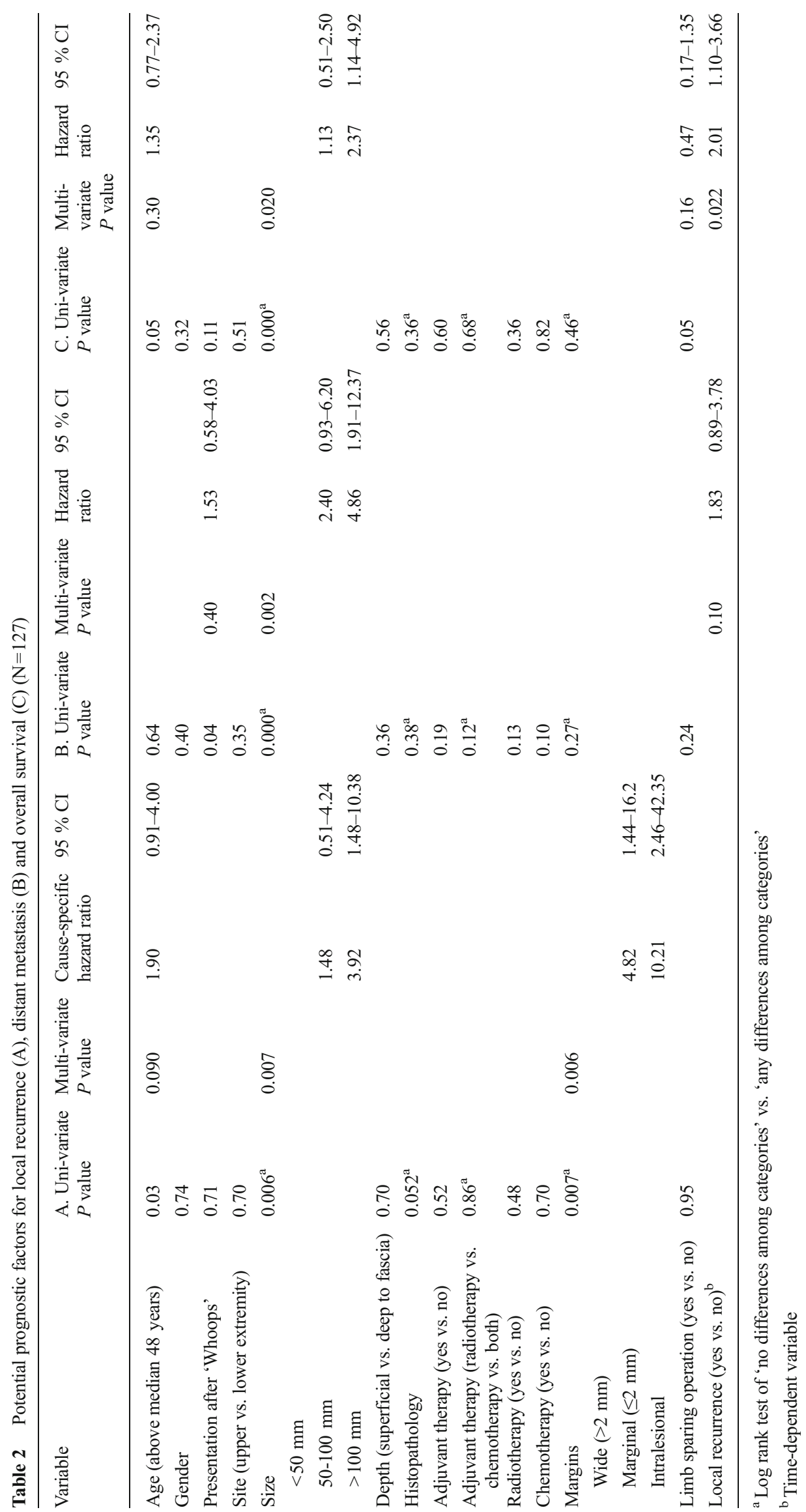




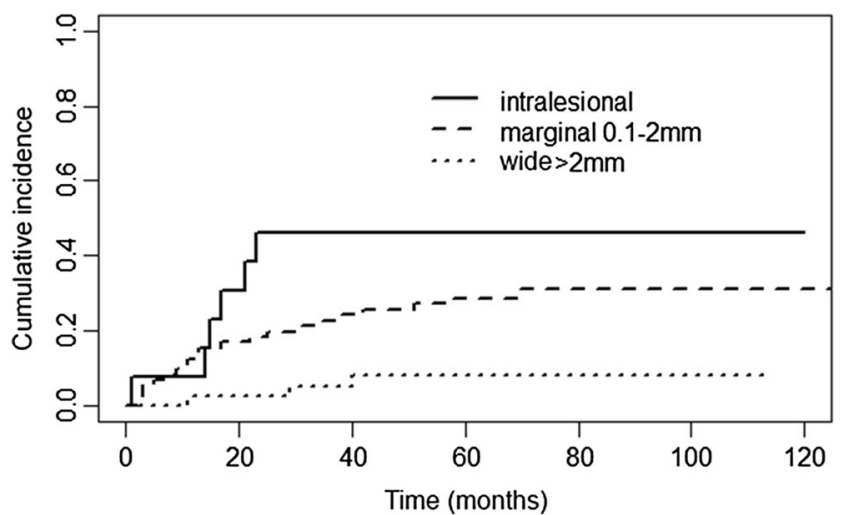

Fig. 2 Cumulative incidence of local recurrence for margins

local recurrence was associated with an increased risk of death, as shown in several studies $[2,13,10,19]$, while others show no association between local recurrence and overall survival $[9,21,22]$. As to the cause of the increased risk we can merely hypothesize between a direct effect of local recurrence on survival and an indirect effect with local recurrence as a marker of high biological tumour aggressiveness. The latter would implicate that the described relationship between local recurrences and overall survival is a result of selection bias. The outcomes of this study reflect the unresolved discussion, with local recurrence as an adverse prognostic factor for survival on one hand, while the lack of a relationship between surgical margins and overall survival argues for local recurrence as a marker of tumour aggressiveness.

When evaluating the effect of margin status, the inconsistencies in definitions of positive margins are an obvious problem. Most investigations classify margin as positive (R1) or negative (R0) margins, because application of Enneking criteria is hampered in retrospective studies. A solely positive or negative status of a margin however, gives no insight into the difference between clear but close margins and wider margins. Exactly this distinction is important in the light of sparing as much tissue as possible for preferable functional outcomes [23]. With improvements in preoperative planning using MRI and the use of (neo) adjuvant therapy, sufficient margins have gotten evermore narrow and the classification of a wide margin as $>2 \mathrm{~mm}$ is more in line with the reality of current deep, high-grade STS resections [6].

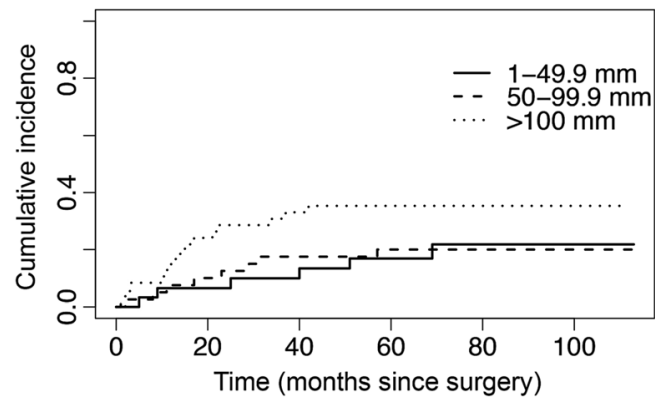

Fig. 3 Cumulative incidence of local recurrence for tumour size
Table 3 Overview of resection margins by tumour size

\begin{tabular}{lllll}
\hline Tumour size & Number, $n$ & Intralesional & Marginal & Wide \\
\hline Small $(<50 \mathrm{~mm})$ & 36 & $4(11 \%)$ & $22(61 \%)$ & $10(28 \%)$ \\
Intermediate & 44 & $6(14 \%)$ & $23(52 \%)$ & $15(34 \%)$ \\
Large $(>100 \mathrm{~mm})$ & 42 & $5(12 \%)$ & $25(60 \%)$ & $12(29 \%)$ \\
\hline
\end{tabular}

Due to the presence of a competing risk (death) precluding an event of interest (local recurrence, metastasis), this study applies the competing risk model. In the presence of competing risks, the Kaplan-Meier method generally overestimates the risk of the event at interest, because it assumes that patients who experienced the competing event are still at risk for the event of interest and thus cumulative probabilities are overestimated [24]. We feel it needs to be noted that many studies, upon which clinical decisions are made, simply overlook the competing risk situation.

Some limitations are present in our study. First of all, due to the low incidence of these sarcomas our sample size is small. This might account for the fact that some expected prognostic factors, such as age or limb sparing procedure, were not significant in our analysis. The second aspect concerns the retrospective nature of the study where selection bias and confounding cannot be excluded. Due to the multicentre aspect of the study the pathologic specimens were neither evaluated by the same pathologist nor were they reviewed to ensure consistency in reporting. Finally, the lack of standardization of adjuvant therapy is a limitation. It has been well established that adjuvant radiotherapy improves local control of STS. The impact on overall survival has however not been established [25]. An attempt to further analyse this aspect was not made in this study. However, there were no significant differences in outcome by any of the adjuvant treatment parameters, except a slight trend of better survival for those receiving adjuvant radiotherapy. In this study $80 \%$ of the patients received adjuvant treatment, thus this minor trend may likely be based on selection bias.

This study shows that tumour size is a consistent adverse prognostic factor for local recurrence, distant metastasis, and overall survival. In addition, our data show a correlation between resection margins and the occurrence of local recurrence. This correlation was not observed for the occurrence of distant metastases or overall survival, while simultaneously a trend of an increased risk of death after local recurrence was noted. The results of this study emphasize once more the need for further convincing evidence of the influence of margins and local recurrence on survival in high-grade ESTS, while stressing the importance of early diagnosis and treatment, with tumour size as the most important prognostic factor. 
Acknowledgments The authors gratefully acknowledge Prof. Pancras Hoogendoorn $(\mathrm{PH})$ and Prof. Judith Bovee (JB) of the Leiden University Medical Center, and Prof. Adrienne Flanagan (AF) of the Royal National Orthopedic Hospital, for their (histo) pathological expertise.

Open Access This article is distributed under the terms of the Creative Commons Attribution License which permits any use, distribution, and reproduction in any medium, provided the original author(s) and the source are credited.

\section{References}

1. Siegel R, Naishadham D, Jemal A (2013) Cancer statistics, 2013. CA-Cancer J Clin 63(1):11-30. doi:10.3322/caac.21166

2. Eilber F, Rosen G, Nelson S, Selch M, Dorey F, Eckardt J, Eilber F (2003) High-grade extremity soft tissue sarcomas: factors predictive of local recurrence and its effect on morbidity and mortality. Ann Surg 237(2):218-226

3. Gronchi A, Casali PG, Mariani L, Miceli R, Fiore M, Lo Vullo S, Bertulli R, Collini P, Lozza L, Olmi P, Rosai J (2005) Status of surgical margins and prognosis in adult soft tissue sarcomas of the extremities: a series of patients treated at a single institution. J Clin Oncol 23(1):96-104. doi:10.1200/JCO.2005.04.160

4. Zagars GK, Ballo MT, Pisters PW, Pollock RE, Patel SR, Benjamin RS, Evans HL (2003) Prognostic factors for patients with localized soft-tissue sarcoma treated with conservation surgery and radiation therapy: an analysis of 1225 patients. Cancer 97(10):2530-2543. doi: 10.1002/cncr.11365

5. Trovik C, Bauer H, Alvegård T, Anderson $\mathrm{H}$, Blomqvist C, Berlin $\mathrm{O}$, Gustafson P, Saeter G, Wallöe A (2000) Surgical margins, local recurrence and metastasis in soft tissue sarcomas: 559 surgicallytreated patients from the Scandinavian Sarcoma Group Register. Eur J Cancer 36(6):710-716

6. Weitz J, Antonescu CR, Brennan MF (2003) Localized extremity soft tissue sarcoma: improved knowledge with unchanged survival over time. J Clin Oncol 21(14):2719-2725. doi:10.1200/JCO.2003.02. 026

7. Italiano A, Le Cesne A, Mendiboure J, Blay JY, Piperno-Neumann S, Chevreau C, Delcambre C, Penel N, Terrier P, Ranchere-Vince D, Lae M, Le Guellec S, Michels JJ, Robin YM, Bellera C, Bonvalot S (2014) Prognostic factors and impact of adjuvant treatments on local and metastatic relapse of soft-tissue sarcoma patients in the competing risks setting. Cancer 120(21):3361-3369. doi:10.1002/cncr. 28885

8. Pisters P, Leung D, Woodruff J, Shi W, Brennan M (1996) Analysis of prognostic factors in 1,041 patients with localized soft tissue sarcomas of the extremities. J Clin Oncol 14(5):1679-1689

9. Tanabe K, Pollock R, Ellis L, Murphy A, Sherman N, Romsdahl M (1994) Influence of surgical margins on outcome in patients with preoperatively irradiated extremity soft tissue sarcomas. Cancer Mar 73(6):1652-1659. doi:10.1002/1097-0142

10. Lewis J, Leung D, Heslin M, Woodruff J, Brennan M (1997) Association of local recurrence with subsequent survival in extremity soft tissue sarcoma. J Clin Oncol 15(2):646-652

11. Stojadinovic A, Leung D, Hoos A, Jaques D, Lewis J, Brennan M (2002) Analysis of the prognostic significance of microscopic margins in 2,084 localized primary adult soft tissue sarcomas. Ann Surg 235(3):424-434

12. Putter H, Fiocco M (2007) Tutorial in biostatistics: Competing risks and multi-state models. Stat Med 26:2389-2430. doi:10.1002/sim. 2712

13. Novais EN, Demiralp B, Alderete J, Larson MC, Rose PS, Sim FH (2010) Do surgical margin and local recurrence influence survival in soft tissue sarcomas? Clin Orthop Relat Res 468(11):3003-3011. doi: 10.1007/s11999-010-1471-9

14. Schemper M, Smith T (1996) A note on quantifying follow-up in studies of failure time. Control Clin Trials 17(4):343-346

15. Team RDC (2008) R: A language and environment for statistical computing. R Foundation for Statistical. http://www.R-project.org. Accessed 10 Feb 2015

16. de Wreede L, Fiocco M, Putter H (2011) mstate: an R package for the analysis of competing rrisks and multi-state models. J Statistical Software 38 (7)

17. O'Donnell PW, Griffin AM, Eward WC, Sternheim A, Catton CN, Chung PW, O'Sullivan B, Ferguson PC, Wunder JS (2014) The effect of the setting of a positive surgical margin in soft tissue sarcoma. Cancer 120(18):2866-2875. doi:10.1002/cncr.28793

18. Friesenbichler J, Leithner A, Maurer-Ertl W, Szkandera J, Sadoghi P, Frings A, Maier A, Andreou D, Windhager R, Tunn PU (2014) Surgical therapy of primary malignant bone tumours and soft tissue sarcomas of the chest wall: a two-institutional experience. Int Orthop 38(6):1235-1240. doi:10.1007/s00264-014-2304-3

19. Gronchi A, Lo Vullo S, Colombo C, Collini P, Stacchiotti S, Mariani L, Fiore M, Casali PG (2010) Extremity soft tissue sarcoma in a series of patients treated at a single institution: local control directly impacts survival. Ann Surg 251(3):506-511. doi:10.1097/SLA. 0b013e3181cf87fa

20. Gronchi A, Verderio P, De Paoli A, Ferraro A, Tendero O, Majo J, Martin J, Comandone A, Grignani G, Pizzamiglio S, Quagliuolo V, Picci P, Frustaci S, Dei Tos AP, Palassini E, Stacchiotti S, Ferrari S, Fiore M, Casali PG (2013) Quality of surgery and neoadjuvant combined therapy in the ISG-GEIS trial on soft tissue sarcomas of limbs and trunk wall. Ann Oncol 24(3):817-823. doi:10.1093/annonc/ mds501

21. Potter D, Kinsella T, Glatstein E, Wesley R, White D, Seipp C, Chang A, Lack E, Costa J, Rosenberg S (1986) High-grade soft tissue sarcomas of the extremities. Cancer 58(1):190-205

22. Rosenberg S, Tepper J, Glatstein E, Costa J, Baker A, Brennan M, DeMoss E, Seipp C, Sindelar W, Sugarbaker P, Wesley R (1982) The treatment of soft-tissue sarcomas of the extremities: prospective randomized evaluations of (1) limb-sparing surgery plus radiation therapy compared with amputation and (2) the role of adjuvant chemotherapy. Ann Surg 196(3):305-315

23. Trovik CS, Skjeldal S, Bauer H, Rydholm A, Jebsen N (2012) Reliability of margin assessment after surgery for extremity soft tissue sarcoma: the SSG experience. Sarcoma 2012:290698. doi:10. $1155 / 2012 / 290698$

24. Biau DJ, Ferguson PC, Chung P, Griffin AM, Catton CN, O'Sullivan B, Wunder JS (2012) Local recurrence of localized soft tissue sarcoma: a new look at old predictors. Cancer 118(23):5867-5877. doi:10. $1002 /$ cncr.27639

25. O'Sullivan B, Davis A, Turcotte R, Bell R, Catton C, Chabot P, Wunder J, Kandel R, Goddard K, Sadura A, Pater J, Zee B (2002) Preoperative versus postoperative radiotherapy in soft-tissue sarcoma of the limbs: a randomised trial. Lancet 359(9325):2235-2241 